\title{
Subventionsbetrug und Verletzung von Cross-Compliance-Vorgaben
}

\author{
Von Dr. Davina Bruhn, Hamburg, Prof. Dr. Jens Bülte, Mannheim*
}

Agrarbetriebe stehen aufgrund der hohen Anforderungen, die sich aus den berechtigten Interessen der Allgemeinheit an einer umweltschonenden Landwirtschaft unter Schutz der Gesundheit von Mensch und Tier sowie anderer ökologischer und kultureller Belange ergeben, unter starkem wirtschaftlichem Druck. Daher ist die Forderung nach einer Subventionierung des Agrarsektors durch die Gesellschaft, die eben diese Rücksichtnahme von der Landwirtschaft verlangt, durchaus berechtigt. Doch steht und fällt dieses Recht auf Gewährung von Subventionen mit der Erfüllung dieser Nachhaltigkeitskriterien für Mindestbedingungen der Tierhaltung, Verwendung von Düngemitteln oder Erhaltung von Landschaftsmerkmalen. Wer Subventionen beantragt, obwohl er seinen Betrieb nicht im Einklang mit den sog. „Grundanforderungen an die Betriebsführung " führt und damit die diese als „anderweitige Verpflichtungen“ oder Cross-Compliance-Pflichten bezeichneten Vorgaben missachtet, verletzt den Grundkonsens der EU-Agrarförderung. Er verliert (zumindest teilweise) seinen Anspruch auf Unterstützung und kann sich zudem wegen Subventionsbetrugs gem. § 264 StGB strafbar machen.

\section{Agrarsubventionen in der Europäischen Union}

Im Haushalt der Europäischen Union sind für das Jahr 2019 für Agrarsubventionen unter dem Haushaltstitel „Nachhaltiges Wachstum: natürliche Ressourcen“ Zahlungen in Höhe von 58,4 Mrd. Euro - das sind 36,1\% der Gesamtmittel der EU - vorgesehen. ${ }^{1}$ Damit stellt die Gemeinsame Agrarpolitik (GAP) den vom Finanzvolumen her zweitwichtigsten Bereich der Politik in der Europäischen Union dar. ${ }^{2}$ Die Agrarförderung erfolgt über zwei Säulen, zum ersten über den europäischen Garantiefonds für Landwirtschaft (EGFL), der marktbezogene Ausgaben und Direktzahlungen an Landwirte beinhaltet, und zum zweiten über den europäischen Landwirtschaftsfonds für die Entwicklung des ländlichen Raums (ELER). ${ }^{3}$ Der Haushaltsansatz für die erste Säule belief sich im Jahr 2019 auf 40,5 Mrd. Euro. ${ }^{4}$ Für diese Direktzahlungen an Agrarunternehmer erhält Deutschland zwischen 2014 und

\footnotetext{
* Dr. Davina Bruhn ist Rechtsanwältin (Hamburg); Prof. Dr. Jens Bülte ist Inhaber des Lehrstuhls für Strafrecht, Strafprozessrecht, Wirtschafts- und Steuerstrafrecht, Universität Mannheim. Die Autoren danken Frau ref. iur. Anna-Lena Dihlmann für sehr wertvolle Anregungen und die kritische Durchsicht des Textes.

1 http://www.europarl.europa.eu/factsheets/de/sheet/106/diefinanzierung-der-gemeinsamen-agrarpolitik (4.11.2019).

${ }^{2} \mathrm{Zu}$ den Zielen der GAP Art. 38 bis 44a AEUV; ferner Groteloth, in: Dombert/Witt (Hrsg.), Münchner Anwaltshandbuch, Agrarrecht, 2. Aufl. 2016 § 27 Rn. 16.

${ }^{3}$ Hierzu und zur historischen Entwicklung der EU-Agrarsubventionen eingehend Booth, in: Dombert/Witt (Fn. 2), § 27 Rn. $61 \mathrm{ff}$.

4 http://www.europarl.europa.eu/factsheets/de/sheet/106/diefinanzierung-der-gemeinsamen-agrarpolitik (4.11.2019).
}

2020 jährlich rund 4,85 Mrd. Euro von der Europäischen Union. ${ }^{5}$ Allein in Nordrhein-Westfalen wickelt die zuständige Landwirtschaftskammer jährlich Fördermittel in Höhe von ca. 600 Millionen Euro ab, die zu $90 \%$ aus dem Haushalt der EU stammen. ${ }^{6}$ Für den hier darzustellenden Zusammenhang ist ausschließlich die Unterstützung von Betrieben durch diese Direktzahlungen relevant.

Seit Ende der 1970er Jahre hatte sich gezeigt, dass staatlich garantierte Annahmepreise für landwirtschaftliche Produkte zu einer unkontrollierten Überproduktion führten, die später als Butterberg und Milchsee bekannt wurden. Daher ging man in den 1990er Jahren dazu über, Stützpreise abzubauen und produktbezogene durch betriebsbezogene Subventionen zu ersetzen. Mit der Agrarreform 2003 begann eine Entkoppelung der Subventionen von den konkreten Produkten, um den Agrarunternehmern mehr unternehmerische Freiräume zu eröffnen und Wettbewerbsverzerrungen abzubauen. ${ }^{7}$ Das führte dazu, dass heute mehr als $98 \%$ der Direktzahlungen an Betriebsinhaber in Deutschland in Form der Betriebsprämie und damit unabhängig von Art und Umfang der landwirtschaftlichen Produktion gewährt werden. ${ }^{8}$ Auf diese Weise sollen Anreize für Überschussproduktionen beseitigt und den Betriebsinhabern die Möglichkeit gegeben werden, sich flexibel und an den betrieblichen Vermarktungsmöglichkeiten orientiert auszurichten. Ferner sollen damit handelsverzerrende Einflüsse auf Preise und die Produktion in außereuropäische Staaten vermieden werden. Seit dem Antragsjahr 2012 und ab dem Haushaltsjahr 2013 wurde ausschließlich die Betriebsprämie gewährt.

Im Jahr 2013 wurden die Agrarsubventionen dahingehend reformiert, dass die bis einschließlich 2014 gewährten Betriebsprämien durch ein differenzierteres System von Direktzahlungen abgelöst wurden. Nunmehr werden ab 2015 flächenabhängige Basisprämien, Umverteilungsprämien und $a b$ 2016 Greeningprämien und gegebenenfalls Junglandwirteprämien ausgezahlt. Insbesondere die Umverteilungsprämie sollte der effektiven Förderung kleiner und mittlerer Betriebe dienen. ${ }^{9}$

Diese Direktzahlungen sind zum einen von der bewirtschafteten Fläche des Betriebs abhängig, zum anderen davon,

\footnotetext{
5 Vgl. hierzu Informationen des Bundesministeriums für Ernährung und Landwirtschaft „Grundzüge der gemeinsamen Agrarpolitik (GAP) und ihre Umsetzung in Deutschland“, abrufbar unter

https://www.bmel.de/DE/Landwirtschaft/Agrarpolitik/ Texte /GAP-NationaleUmsetzung.html (4.11.2019).

${ }^{6}$ Angabe der Landesregierung NRW, Landtag NRW Drs. 17/ 3350, S. 2.

${ }^{7}$ Vgl. zum Begriff der Entkopplung Booth (Fn. 3), Rn. 78.

${ }^{8} \mathrm{Vgl}$. hierzu Informationen der Bundesanstalt für Landwirtschaft und Ernährung „Gemeinsame Agrarpolitik und EUAgrarausgaben", abrufbar unter

https://www.agrar-fischerei-

zahlungen.de/agrar foerderung.html (4.11.2019).

${ }^{9}$ Vgl. Booth (Fn. 3), Rn. 96 ff.
} 
dass der Unternehmer die unionsrechtlichen Vorgaben des Natur-, Umwelt-, Tier- und Verbraucherschutzes sowie zur Erhaltung der Flächen in gutem landwirtschaftlichen und ökologischen Zustand, etwa zur Erosionsvermeidung oder zum Schutz von Grund- und Oberflächengewässern, einhält. Weil diese Vorgaben zur Nachhaltigkeit nicht im unmittelbaren Zusammenhang mit der Produktion selbst stehen müssen, spricht man insofern von Cross-Compliance-Vorgaben (CCVorgaben). ${ }^{10}$ Die Direktzahlungen sollen also nicht nur die wirtschaftliche Existenz der Agrarunternehmen und die Ernährung der Bevölkerung sichern, sondern auch einen Beitrag zum Erhalt landeskultureller Werte, nachhaltiger Landwirtschaft und verantwortungsvoller Tierhaltung sowie zum Verbraucherschutz leisten. ${ }^{11}$

\section{Regeln der Cross Compliance und Sanktionsfolgen bei Verstößen}

Da die Europäische Union im internationalen Vergleich hohe Standards bei dem Betrieb der Landwirtschaft setzt und dadurch Wettbewerbsnachteile entstehen können, sollen die Direktzahlungen aus dem EU-Agrarhaushalt unter anderem dem Ausgleich der höheren Produktionskosten dienen. Der Verbindung zwischen den Subventionen und den damit ausgeglichenen Nachteilen durch besondere Schutzstandards soll durch die Auflagenbindung, die als CC bezeichnet wird, rechtliche Verbindlichkeit und praktische Effektivität erlangen. In der Konsequenz dieses Systems dürfen EU-Agrarsubventionen nur gewährt und belassen werden, wenn die entsprechenden Standards eingehalten werden.

\section{Bedeutung und Wirkung der Cross-Compliance-Vorgaben}

Den CC-Verpflichtungen unterliegen grundsätzlich alle Betriebe - mit Ausnahme von Kleinerzeugern -, die Direktzahlungen aus der ersten Säule erhalten, aber auch solche, denen Zahlungen für flächen- und tierbezogene Maßnahmen der zweiten Säule oder Umstrukturierungsbeihilfen im Weinbereich zugutekommen. Die Einzelheiten der Vorgaben aus der CC regelt zunächst die Verordnung (EU) Nr. 1306/2013 ${ }^{12}$, die auf weitere Rechtsakte der Europäischen Union wie die Delegierte Verordnung (EU) Nr. 640/2014 ${ }^{13}$ und ergänzende Vorschriften der Mitgliedstaaten zurückgreift. Für Deutsch-

\footnotetext{
${ }^{10} \mathrm{Vgl}$. Booth (Fn. 3), Rn. 79, $163 \mathrm{ff}$.

${ }^{11}$ Vgl. hierzu Informationen der Bundesanstalt für Landwirtschaft und Ernährung: „Gemeinsame Agrarpolitik und EUAgrarausgaben", abrufbar unter

https://www.agrar-fischerei-

zahlungen.de/agrar foerderung.html (4.11.2019).

${ }^{12} \mathrm{VO}$ (EU) des europäischen Parlaments und des Rates vom 17.12.2013 über die Finanzierung, die Verwaltung und das Kontrollsystem der Gemeinsamen Agrarpolitik, ABl. EU v. 20.12.2013, Nr. L 307 40/549.

${ }^{13}$ Delegierte Verordnung (EU) Nr. 640/2014 der Kommission vom 11.3.2014 zur Ergänzung der Verordnung (EU) Nr. 1306/2013 des Europäischen Parlaments und des Rates, ABl. EU v. 20.6.2014, Nr. L181/48.
}

land gelten das Agrarzahlungen-Verpflichtungengesetz und die Agrarzahlungen-Verpflichtungenverordnung. ${ }^{14}$

$\mathrm{Zu}$ den Voraussetzungen aus der CC-Pflicht, die ein Betriebsinhaber erfüllen muss, wenn er Direktzahlungen erhält, gehören zunächst die sog. Grundanforderungen an die Betriebsführung (GAB). Sie umfassen die wichtigsten Regeln aus den Bereichen Umweltschutz, Lebens- und Futtermittelsicherheit, Tierkennzeichnung und -registrierung, Tierseuchenbekämpfung, Pflanzenschutzmitteleinsatz und Tierschutz. Sie ergeben sich aus insgesamt 13 europäischen Rechtsakten, gegebenenfalls den Umsetzungsakten der Mitgliedstaaten und gelten grundsätzlich auch unabhängig von der Subventionsgewährung. Ferner sind die Standards zur Erhaltung landwirtschaftlicher Flächen in ,gutem landwirtschaftlichen und ökologischen Zustand" (GLÖZ) ${ }^{15}$ einzuhalten. Sie betreffen insbesondere die Verminderung von Bodenerosion, die Verhinderung der Beseitigung von Landschaftselementen, der Begrünung von nicht zur Erzeugung genutzten Flächen sowie den Gewässerschutz. Die Vorgaben der GAB und der GLÖZ sind im Anhang II zur VO (EU) Nr. 1306/2013 aufgeführt. ${ }^{16}$ Dabei legt das Unionsrecht einen gesamtbetrieblichen Ansatz zugrunde, so dass die CCPflichten nicht nur für die landwirtschaftlichen Flächen einzuhalten sind, für die konkret Beihilfen beantragt wurden, sondern im gesamten landwirtschaftlichen Betrieb. ${ }^{17}$

In der VO (EU) Nr. 1307/2013 ${ }^{18}$ und der Delegierten VO (EU) Nr. 639/2014 ${ }^{19}$ finden sich Regelungen über die Direktzahlungen an Agrarunternehmer. Art. 21 ff. VO (EU) 1307/ 2013 legt fest, unter welchen Bedingungen der Betriebsinhaber die Basisprämienregelung - also die flächenabhängige Direktzahlung - in Anspruch nehmen darf. Voraussetzung dafür ist, dass einem aktiven Betriebsinhaber (vgl. Art. 9 VO [EU] Nr. 1307/2013; Art. 10 ff. VO [EU] Nr. 639/2014) ${ }^{20}$ für förderfähige Betriebsflächen sog. Zahlungsansprüche zugewiesen worden sind (vgl. insb. Art. 14 ff. VO [EU] Nr. 639/ 2014). ${ }^{21}$ Ferner gelten das Direktzahlungendurchführungsgeset $^{22}$ und die Direktzahlungendurchführungsverordnung. ${ }^{23}$

\section{Verwaltungssanktionen bei Verletzung der Cross- Compliance-Vorschriften}

Verletzt ein Betriebsinhaber, der Direktzahlungen erhält, seine Pflicht zur Einhaltung der CC-Vorgaben, weil er etwa

\footnotetext{
${ }^{14} \mathrm{Vgl}$. weiterhin Booth (Fn. 3), Rn. 88 ff.

${ }^{15}$ Vgl. Booth (Fn. 3), Rn. 182.

${ }^{16}$ Vgl. hierzu Booth (Fn. 3), Rn. 173.

${ }^{17}$ EuGH, Urt. v. 7.8.2018 - C-435/17.

18 VO (EU) Nr. 1307/2013 v. 17.12.2013, ABl. EU v. 20.12.2013, Nr. L 347/608.

${ }^{19}$ Delegierte VO (EU) Nr. 639/2014 v. 11.3.2014, AB1. EU v. 20.6.2014, Nr. L 181/1.

${ }^{20} \mathrm{Vgl}$. Booth (Fn. 3), Rn. 105 ff.

${ }^{21}$ Zum Antrags- und Zuweisungsverfahren von Zahlungsansprüchen Booth (Fn. 3), Rn. 109 ff.

${ }^{22}$ Gesetz v. 9.7.2014 (BGBl. I, S. 897), i.d.F. v. 21.10.2016 (BGB1. I, S. 2370).

${ }^{23}$ VO v. 3.11.2014 (BGBl. I, S. 1690), i.d.F. v. 22.2.2019 (BGBl. I S. 170).
} 
gegen Vorschriften zum Umgang mit wassergefährdenden Stoffen durch ablaufenden Silagesickersaft ${ }^{24}$ (GLÖZ 3) oder mit Tierarzneimitteln verstößt (Art. 17 Abs. 1 der BasisVO (VO [EU] Nr. 178/2002; GAB 4), ${ }^{25}$ so ordnen Art. 91 ff. VO (EU) Nr. 1306/2013 die Kürzung der Subvention im Wege einer „Verwaltungssanktion“ an. Diese Verwaltungssanktionen haben anspruchsvernichtende Wirkung. Eine bereits bewilligte Subvention darf nicht mehr ausgezahlt werden, soweit sie gekürzt wurde. ${ }^{26}$

Art. 91 Abs. 1 VO (EU) Nr. 1306/2013 bestimmt insofern zunächst, dass gegen einen Empfänger von Direktzahlungen (Begünstigter), der die CC-Vorschriften gemäß Art. 93 VO (EU) Nr. 1306/2013 nicht einhält, eine Verwaltungssanktion $\mathrm{zu}$ verhängen ist. Voraussetzung dieser zwingenden Rechtsfolge ist nach Art. 91 Abs. 2 VO (EU) Nr. 1306/2013, dass der Verstoß dem Begünstigten als Ergebnis seiner Handlung oder Unterlassung anzulasten ist ${ }^{27}$ und seine landwirtschaftliche Tätigkeit oder die Fläche seines Betriebs betrifft. Die sanktionsbewehrten CC-Pflichten sind durch Art. 93 Abs. 1 VO (EU) Nr. 1306/2013 bestimmt, der auf Anhang II der Verordnung verweist. Dort wird auf bestimmte EU-Verordnungen sowie auf Richtlinien verwiesen. Art 93 Abs. 2 VO (EU) Nr. 1306/2013 bestimmt, dass im Falle von Mindestvorgaben, die in Richtlinien festgelegt sind, die jeweiligen nationalen Umsetzungsakte (z.B. für GAB 11-13 die deutsche TierSchNutztV) maßgeblich sind.

Die Anwendung und Berechnung der Verwaltungssanktionen regeln Art. 97 ff. VO (EU) Nr. 1306/2013. Zunächst bestimmt Art. 97 Abs. 1 VO (EU) Nr. 1306/2013, dass die Nichterfüllung der CC-Vorgaben zu irgendeinem Zeitpunkt im Kalenderjahr, für das die Direktzahlungen gewährt werden, die Sanktion zur Folge hat. Nach Art. 99 Abs. 1 VO (EU) Nr. 1306/2013 erfolgt die Sanktionierung durch die Kürzung oder Streichung des Gesamtbetrags der Direktzahlungen für das Jahr, in dem der Verstoß festgestellt wurde. Bei der Berechnung der Kürzungen sind nach Art. 99 UAbs. 1 VO (EU) Nr. 1306/2013 die Schwere, das Ausmaß, die Dauer und ggf. eine wiederholte Begehung des Verstoßes zu berücksichtigen. Hierzu finden sich in Art. 38 Delegierte VO (EU) Nr. 640/2014 nähere Regelungen.

Nach Art. 99 Abs. 2 VO (EU) Nr. 1306/2013 beträgt die Kürzung - für jeden Verstoß - bei Fahrlässigkeit höchstens $5 \%$ des Gesamtbetrages, im Wiederholungsfall $15 \%$. Art. 39 Abs. 1 Delegierte VO (EU) Nr. 640/2014 gibt als regelmäßigen Kürzungssatz $3 \%$ an. Art. 39 Abs. 4 Delegierte VO (EU) Nr. 640/2014 bestimmt ferner, dass der Kürzungssatz in jedem Wiederholungsfall jeweils zu verdreifachen ist. Bei Erreichen des Höchstsatzes von $15 \%$ weist die Behörde zudem darauf hin, dass bei jedem weiteren Verstoß dieser Art von einer vorsätzlichen Begehung ausgegangen wird. Bei

\footnotetext{
${ }^{24}$ Vgl. VG Regensburg, Urt. v. 21.3.2019 - RN 5 K 17.1365.

${ }^{25}$ Vgl. VG München, Urt. v. 12.7.2018 - M 12 K 17.5704.

${ }^{26}$ Booth (Fn. 3), Rn. 168.

${ }^{27}$ Zur Zurechnung von Verstößen EuGH, Urt. v. 27.2.2014 C-396/12 (Van der Ham), Rn. 48 ff. = AUR 2014, 147 (149); EuGH, Urt. v. 13.12.2012 - C-11/12 (Maatschap), Rn. 36 ff. = NVwZ 2013, 134 (135 f.).
}

vorsätzlichen Verstößen sieht Art. 99 Abs. 3 VO (EU) Nr. 1306/2013 eine Kürzung von mindestens $20 \%$ bis zum völligen Ausschluss von Direktzahlungen, auch für mehrere Jahre vor. Art. 40 Delegierte VO (EU) Nr. 640/2014 regelt die Details der Sanktionsverhängung.

Mit Blick auf die Verwaltungssanktion muss berücksichtigt werden, dass der Vorsatzbegriff der VO (EU) Nr. 1306/ 2013 unionsrechtlich autonom auszulegen ist. Dennoch hat die Vierte Kammer des EuGH den Vorsatzbegriff von Art. 67 Abs. 1 VO (EG) 796/2004 - der Vorgängernorm zu Art 99 VO (EU) Nr. 1306/2013 - ähnlich wie im deutschen Strafrecht ausgelegt. ${ }^{28}$ Das Haftungssystem der Subventionskürzung habe keinen (rein) objektiven Charakter. Der „Begriff des ,vorsätzlichen Verstoßes' [sei] dahin auszulegen, dass es hierfür erforderlich ist, dass der durch die Beihilfe Begünstigte gegen die Vorschriften über die anderweitigen Verpflichtungen verstößt und diesen Verstoß entweder bewusst herbeiführt oder - ohne dass er ein solches Ziel verfolgt - die Möglichkeit eines derartigen Verstoßes billigend in Kauf nimmt." Die Anordnung widerlegbarer Beweisvermutungen im Recht der Mitgliedstaaten zur Feststellung des Vorsatzes hat die Kammer allerdings ausdrücklich nicht ausgeschlossen. ${ }^{29}$

\section{Bewilligungs- und Sanktionspraxis}

Aus der Rechtsprechung der Verwaltungsgerichte und den Informationsbroschüren der Behörden lässt sich erahnen, wie die Behördenpraxis der Bewilligung und Sanktionierung aussieht: Die Behörde stellt aufgrund des Antrags des Unternehmers die grundsätzliche Förderungswürdigkeit fest und bewilligt die Direktzahlungen. Dabei wird grundsätzlich von der Richtigkeit der Angaben des Antragstellers und der vorgelegten Unterlagen ausgegangen. In einer statistisch und aufgrund von Risikoprofilen festgelegten Zahl von Betrieben findet dann vor der Auszahlung der Subvention zum Jahresende eine zufällige Routinekontrolle statt. Führt diese zur Feststellung von CC-Verstößen, so erfolgt eine Kürzung der bewilligten Subvention durch Bescheid und die Auszahlung am Jahresende wird verringert. Zudem kann es zu anlassbedingten Kontrollen aufgrund von Anzeigen (sog. CrossCheck) oder bei Fachkontrollen in anderem Zusammenhang kommen. Wird bei einer solchen Kontrolle ein CC-Verstoß festgestellt, kommt es ebenfalls zu einer Kürzung des auszuzahlenden Subventionsbetrages.

\section{Strafrechtliche Einordnung als Subventionsbetrug}

Wird ein begünstigter Agrarunternehmer mit einer Verwaltungssanktion belegt, so stellt sich die Frage, ob die Antragstellung trotz Verletzung der CC-Vorgaben zusätzlich zur Subventionskürzung strafrechtliche Folgen nach nationalem Recht haben kann. In Art. 3 Delegierte VO (EU) Nr. 640/ 2014 ist die Möglichkeit einer strafrechtlichen Sanktion ausdrücklich angelegt: „Die Anwendung der in dieser Verord-

28 EuGH, Urt. v. 27.2.2014 - C-396/12 (Van der Ham), Rn. 27 ff. = AUR 2014, 147 (148).

${ }^{29}$ EuGH, Urt. v. 27.2.2014 - C-396/12 (Van der Ham), Rn. 41 = AUR 2014, 147 (148 f.). 
nung vorgesehenen Verwaltungssanktionen und Ablehnung oder Rücknahme der Beihilfe erfolgen unbeschadet der Anwendung strafrechtlicher Sanktionen, soweit diese nach nationalem Recht vorgesehen sind.“

Damit ist natürlich noch nicht gesagt, dass dieses Verhalten auch deutschen Strafvorschriften unterfällt. In Betracht käme jedoch insbesondere eine Strafbarkeit nach $§ 264$ StGB, wenn der Betriebsinhaber einen Antrag auf Gewährung der Direktzahlungen in Form der Basisprämie stellt, dabei zutreffende Angaben über seinen Betrieb macht, aber bei Antragstellung bereits weiß, dass er die CC-Vorgaben nicht eingehalten hat oder einhalten wird. Diese Fallgestaltung dürfte aufgrund der Besonderheiten des Antragsverfahrens nicht selten sein. Wird der Antrag im Mai des Jahres gestellt, für das die Subvention bewilligt werden soll, so ist im Antragszeitpunkt oftmals bereits bekannt, ob in den ersten Monaten des laufenden Jahres CC-Verstöße begangen worden sind.

Diese Ausgangssituation wirft eine Reihe von Detailfragen auf, weil zum einen $\S 264$ StGB ein hochkomplexer Tatbestand ist, der eine Vielzahl von Rechtsproblemen bereitet. Zum anderen ist die Sanktionierung von Missbrauchshandlungen im Kontext von EU-Haushaltsmitteln stets im Lichte des Unionsrechts, insbesondere Art. 325 AEUV und der Richtlinie (EU) 2017/1371 vom 5.7.2017 über die strafrechtliche Bekämpfung von gegen die finanziellen Interessen der Europäischen Union gerichtetem Betrug zu betrachten. Der effektive Schutz des EU-Haushalts ist eine Maxime, die bei der Auslegung von Strafvorschriften mit Blick auf den unionsrechtlichen Anwendungsvorrang - natürlich in den Grenzen der Art. 103 Abs. 2 GG und Art. 49 EU-GRCh stets zu beachten ist. ${ }^{30}$

Macht sich also ein Unternehmer nach $§ 264$ StGB strafbar, wenn er Direktzahlungen beantragt, obwohl er weiß, dass er z.B. die Vorgaben aus Art. 3 und 4 der Richtlinie 2008/ 120/EG des Rates über Mindestanforderungen für den Schutz von Schweinen nicht einhält bzw. nicht einhalten wird, weil die baulichen Verhältnisse der Haltungseinrichtungen, die Höchstlärmbelastung, die Mindestvorgaben für Licht oder den Toleranzrahmen für die Temperatur nicht hergeben etc.? Führt also jeder bei der Beantragung bekannte CC-Verstoß durch Verletzung der GAB bei Mängeln in der Tierhaltung, Düngemittelverwendung etc., zu einer Strafbarkeit nach $\S 264$ StGB?

Die Annahme einer solchen Strafbarkeit liegt nahe, denn in einem solchen Fall verschweigt ein Betriebsinhaber bei der Geltendmachung eines Anspruchs auf eine Subvention einen Umstand, der zwangsläufig wegen des vorsätzlichen CC-Verstoßes zu einer Kürzung der Subvention in Höhe von mindestens $20 \%$ führen würde. Doch ist die Strafbarkeit wegen $\S 264$ StGB an strenge Voraussetzungen geknüpft, so dass die Vorschrift nur einen engen Bereich von Täuschungshandlungen im Vorfeld des $§ 263$ StGB abdeckt.

\footnotetext{
${ }^{30}$ Vgl. nur EuGH, Urt. v. 5.12.2017 - C-42/17 (MAS \&
} MB), Rn. 39 ff. = NJW 2018, 217 (218) m.w.N.

\section{Direktzahlungen als Subventionen im Sinne von $\S 264$ St $G B$}

Strafbar ist der Subventionsbetrug nach $\S 264$ StGB naturgemäß nur dann, wenn sich die Tathandlung auf die Erlangung einer Subvention nach $\S 264$ Abs. 8 StGB richtet und im Rahmen eines Subventionsverfahrens begangen wird. Beides ist hier gegeben, weil erstens die Direktzahlungen Leistungen aus öffentlichen Mitteln nach dem Recht der Europäischen Union sind, die ohne marktmäßige Gegenleistung gewährt werden. Damit handelt es sich um Subventionen nach $\S 264$ Abs. 8 Nr. 2 StGB. Zweitens werden in der zugrundeliegenden Konstellation für die Gewährung der Direktzahlungen relevante Tatsachen im Rahmen des Subventionsverfahrens und nicht nur bei Gelegenheit von Vorerkundigungen nicht offengelegt.

\section{Subventionserhebliche Tatsache i.S.v. § 264 Abs. 9 StGB}

Der Subventionsbetrug wird durch eine der spezifischen Tathandlungen begangen, die in $\S 264$ Abs. 1 Nr. 1 bis 4 StGB umschrieben sind. Vorliegend kommt vornehmlich eine Tathandlung nach Nr. 1 in Betracht. Tatbestandlich handelt danach, wer gegenüber einer „für die Bewilligung einer Subvention zuständigen Behörde oder einer anderen in das Subventionsverfahren eingeschalteten Stelle oder Person (Subventionsgeber) über subventionserhebliche Tatsachen für sich oder einen anderen unrichtige oder unvollständige Angaben macht, die für ihn oder den anderen vorteilhaft sind“". Der Täter muss also über eine subventionserhebliche Tatsache täuschen, um eine Tat nach $\S 264$ Abs. 1 Nr. 1 StGB zu begehen.

Durch den Begriff der subventionserheblichen Tatsache werden die Tathandlungen des $\S 264$ Abs. 1 Nr. 1, 3 und 4 StGB auf Angaben über spezifische Umstände reduziert und die Vorverlagerung des strafrechtlichen Täuschungsschutzes auf das kriminalpolitisch Notwendigste begrenzt. Nur die Täuschung über subventionserhebliche Tatsachen ist nach $\S 264$ Abs. 1 Nr. 1 StGB strafbar. Dabei ist der Tatsachenbegriff wie in $\S 263$ StGB zu verstehen, als Vorgänge oder Zustände der Vergangenheit oder Gegenwart, die objektiv bestimmt und dem Beweis zugänglich sind. ${ }^{31}$

Ob Tatsachen subventionserheblich sind, bestimmt $§ 264$ Abs. 9 StGB. Aus seiner Legaldefinition wird deutlich, dass die Subventionserheblichkeit grundsätzlich nicht aus der sachlichen Bedeutung einer bestimmten Tatsache für die Subvention abgeleitet werden darf, ${ }^{32}$ sondern allein durch die konstitutive Erklärung des Gesetzes selbst oder durch den Subventionsgeber aufgrund Gesetzes entsteht.

\footnotetext{
${ }^{31} \mathrm{Zu} \S 263$ StGB vgl. BGH, Urt. v. 14.3.2019 - 4 StR 426/18 = NJW 2019, 1759; Dannecker, in Graf/Jäger/Wittig (Hrsg.), Wirtschafts- und Steuerstrafrecht, 2. Aufl. 2017, § 263 Rn. 14.

${ }^{32}$ Vgl. nur BGH, Urt. v. 11.11.1998 - 3 StR 101/98 = BGHSt 44, 233 (239).
} 
a) Formelle Subventionserheblichkeit aufgrund der Bestimmung durch Gesetz oder aufgrund eines Gesetzes

Daher sind Tatsachen „subventionserheblich“, wenn sie „durch Gesetz oder auf Grund eines Gesetzes von dem Subventionsgeber als subventionserheblich bezeichnet sind" (§ 264 Abs. 9 Nr. 1).

Damit ist zum einen der Fall erfasst, in dem das materielle Gesetz - eine Rechtsverordnung ist also ausreichend, nicht aber Verwaltungsvorschriften, Verwaltungsrichtlinien o.a.. ${ }^{33}$ selbst die Subventionserheblichkeit feststellt ( $\$ 264$ Abs. 9 Nr. 1 Var. 1 StGB). Dabei muss nicht der Begriff „subventionserheblich“ verwendet, aber unmissverständlich festgestellt werden, dass das Gewähren oder Belassen der Subvention von diesem Umstand abhängt. Zum anderen ist eine Tatsache subventionserheblich, wenn sie von der Behörde als subventionserheblich bezeichnet wird und dies auf der Grundlage eines Gesetzes (z.B. des SubvG) erfolgt.

Danach hat die für die Vergabe zuständige Stelle (Subventionsgeber) dem Subventionsnehmer gegenüber die Tatsachen als ,subventionserheblich im Sinne des § 264 Strafgesetzbuch zu bezeichnen, die 1. nach dem Subventionszweck, 2. den Rechtsvorschriften, Verwaltungsvorschriften und Richtlinien über die Subventionsvergabe sowie 3. den sonstigen Vergabevoraussetzungen für die Bewilligung, Gewährung, Rückforderung, Weitergewährung oder das Belassen einer Subvention oder eines Subventionsvorteils erheblich sind“.

Der $\mathrm{BGH}^{34}$ hat die verfassungsrechtliche Bedeutung dieser Pflicht des Subventionsgebers zur exakten Bezeichnung der subventionserheblichen Tatsachen betont. Der Antragsteller müsse klar erkennen können, welche Umstände (strafrechtlich) relevante Vergabevoraussetzungen darstellen. ${ }^{35}$ Die besondere Deutlichkeit sei geboten, weil die Strafbarkeit durch $\S 264$ StGB erheblich vorverlagert sei, indem bereits die Täuschungshandlung ohne Schadenseintritt pönalisiert werde und auch Leichtfertigkeit strafbar sei. Es reiche nicht aus, den potenziellen Täter ,generell darauf hinzuweisen, dass die Behörde an seinen Angaben interessiert ist". Er soll vielmehr konkret auf bestimmte Tatsachen hingewiesen werden, ,deren richtige und vollständige Beurteilung die zweckentsprechende Verwendung öffentlicher Mittel sicherstellen soll“". Zudem müssten auch der Subventionsgeber und ggf. die Strafverfolgungsbehörden in die Lage versetzt werden, rasch und eindeutig festzustellen, ob strafbare Täuschungen begangen wurden. Daher seien unmissverständliche und auf den konkreten Fall bezogene Angaben unabdingbar. Es reiche nicht aus, wenn sich die Subventionserheblichkeit „lediglich aus dem Zusammenhang ergibt“, ,,pauschal oder formelhaft bezeichnet" oder sich die Bezeichnung der subventionserheblichen Tatsachen in der Wiederholung der in $\S 264$ Abs. 9 Nr. 2 StGB aufgeführten abstrakten Umstände erschöpft. ${ }^{36}$

\footnotetext{
33 BT-Drs. 7/3411, S. 28; BGH, Urt. v. 11.11.1998 - 3 StR 101/98 = BGHSt 44, 233 (237).

${ }^{34}$ BGH, Urt. v. 11.11.1998 - 3 StR 101/98 = BGHSt 44, 233 (238).

${ }^{35}$ Vgl. auch BT-Drs. 7/3441, S. 28.

${ }^{36}$ BGH, Urt. v. 11.11.1998 - 3 StR 101/98 = BGHSt 44, 233 (238).
}

Diese Anforderungen gelten sowohl für die Bestimmung der Subventionserheblichkeit durch das Gesetz, als auch aufgrund eines Gesetzes nach $\S 264$ Abs. 9 Nr. 1 Var. 2 StGB. § 2 SubvG beinhaltet zwar eine solche Regelung, die den Subventionsgeber einerseits zur ausdrücklichen Bezeichnung von Subventionen als (strafrechtlich) erheblich verpflichtet, ${ }^{37}$ andererseits aber auch die Rechtsgrundlage für die Bestimmung von Tatsachen als subventionserheblich i.S.v. $\S 264$ Abs. 9 Nr. 1 Var. 2 StGB darstellt. Ob § 2 SubvG allerdings auf Subventionen anwendbar ist, die nach Unionsrecht vergeben werden oder nur auf Subventionen aus dem Haushalt des Bundes oder der Länder - soweit die jeweiligen Landesgesetze auf das SubvG verweisen (vgl. § 1 LSubvG NRW) - ist zweifelhaft.

Aus den Materialien zum Entwurf des 1. Gesetzes zur Bekämpfung der Wirtschaftskriminalität ergibt sich nur, dass das SubvG nicht anwendbar sein soll, soweit die Subventionen von Stellen der Europäischen Gemeinschaften unmittelbar verwaltet und vergeben werden. Direktzahlungen wie die Basisprämie und Greeningprämie sind Subventionen, deren Vergabe auf einem Haushaltsansatz der europäischen Union beruht und durch Verordnungen des Unionsrechts und Detailregelungen des nationalen Rechts geregelt ist. Daher könnte man hier annehmen, dass $\S 2$ SubvG keine Anwendung findet.

In der Begründung zum Entwurf des $\S 1$ SubvG vom 1.4.1975 heißt es zur Anwendung des damals neuen Gesetzes auf EG-Subventionen, der Bundesgesetzgeber sei ,in der Lage, eine Leistung nach dem Gemeinschaftsrecht als Subventionen im Sinne des neuen $\S 264$ StGB zu bezeichnen“. 38 Jedoch könne zweifelhaft sein, ob die $\S \S 4$ bis 7 SubvG auch bei Leistungen nach dem Gemeinschaftsrecht greifen, weil Mitgliedstaaten nicht befugt seien, ,in einem Bereich normative Regeln zu erlassen, in welchem die Rechtsetzungsbefugnis auf die Gemeinschaft übertragen ist".

Das lässt den Umkehrschluss zu, $\S \S 1$ bis 3 SubvG könnten auch für Subventionen gelten, die nach dem Unionsrecht vergeben werden, für deren Bewilligung, Gewährung etc. aber nationale Behörden zuständig sind. Davon gehen wohl auch die zuständigen Landesbehörden aus, wie sich etwa aus dem Mantelbogen des Sammelantrages der Landwirtschaftskammer NRW für das Jahr 2019 ergibt. Der Antragsteller hat in diesem Formular nach Ziffer 13.12 zu versichern, ihm sei bekannt, „dass, alle Angaben dieses Antrages, von denen die Bewilligung, Gewährung, Weitergewährung oder das Belassen der Zuwendung abhängig sind, subventionserheblich im Sinne des $§ 264$ Strafgesetzbuch (Erstes Gesetz zur Bekämpfung der Wirtschaftskriminalität 1. WiKG) in Verbindung mit $\S 1$ des Gesetzes über die Vergabe von Subventionen nach Landesrecht (Landessubventionsgesetz) vom 24.03.1977 sind und bei entsprechender Nichteinhaltung strafrechtlich verfolgt werden $[\ldots]^{\text {“. }}$.

Dieser Hinweis dürfte aber wohl nicht ausreichen, um den Antragsteller mit der vom BGH postulierten Eindeutigkeit

\footnotetext{
${ }^{37}$ BGH, Urt. v. 11.11.1998 - 3 StR 101/98 = BGHSt 44, 233 (241).

${ }^{38}$ BT-Drs. 7/3441, S. 42.
} 
darauf hinzuweisen, welche Angaben als spezifisch subventionserheblich anzusehen sind und welche nicht. Dies gilt auch, wenn der Antragsteller auf dem Antragsformular zum Abschluss erklärt, dass die Angaben und Erklärungen der einzelnen angekreuzten Anlagen Bestandteil des Antrages sind und er die Verpflichtungen zu den einzelnen Antragsunterlagern anerkennt.

Die „dritte Seite“ des o.g. Mantelbogens enthält ferner Verpflichtungserklärungen (Ziffer 11), Einverständnis- und Anerkenntniserklärungen (Ziffer 12) und Wissenserklärungen (Ziffer 13). Der Antragsteller erklärt sich u.a. bereit zur Bereitstellung von Unterlagen (Ziffer 11.2.), zur Bezeichnung von bewirtschafteten Flurstücken (sog. Schläge, Ziffer 11.3.), zur unverzüglichen Mitteilung von subventionserheblichen Abweichungen, Änderungen oder Wechsel und alle Tatsachen (Ziffer 11.4) und zur Aufbewahrung von Unterlagen (Ziffer 11.5).

Unter Ziffer 12 erklärt er u.a., dass er die geltenden Bedingungen für die Gewährung der beantragten Beihilfe zur Kenntnis genommen hat und anerkennt (Ziffer 12.1), dass er keine weiteren Direktzahlungen beantragt hat (Ziffer 12.2), keine Umgehungskonstruktionen geschaffen hat (Ziffer 12.3), die Angaben im Antrag zutreffend sind (Ziffer 12.6) und dass der Antragsteller die „Informationen über die anderweitigen Verpflichtungen (Cross Compliance-Broschüre) für 2019 von der EU-Zahlstelle [...] erhalten" hat und ihm der Inhalt bekannt ist.

Schließlich werden unter Ziffer 13 weitere 17 Erklärungen darüber abgegeben, was dem Antragsteller ansonsten noch alles bekannt sei. Dazu gehört unter Ziffer 13.4 der Inhalt der für die Direktzahlungen relevanten Verordnungen des Europäischen Parlaments und des Rates (insbesondere Nr. 1035/2013, 1306/2013, 1307/2013) und die konkretisierenden Delegierten Verordnungen der Kommission (Nr. 639641/2014 und 807-809/2014).

Diese Verweise auf eine Vielzahl nicht exakt genannter Tatsachen, die der Antragsteller erst noch aus den Rechtsgrundlagen heraussuchen muss, dürften kaum ausreichen, um jede der Angaben im Antragsformular (z.B. auch im Formular angegebene Mobilfunk- oder Faxnummer o.ä.) als subventionserhebliche Tatsache im Sinne von $\S 264$ Abs. 9 Nr. 1 Var. 2 StGB zu klassifizieren. Letztlich kann diese Frage aber ebenso dahinstehen wie die Anwendbarkeit von $\S 2$ SubvG, da insoweit $\S 264$ Abs. 9 Nr. 2 StGB zur Anwendung kommt.

\section{b) Materielle Subventionserheblichkeit aufgrund gesetzlich bestimmter Relevanz.}

Nach dieser Vorschrift gelten „,neben den formell als subventionserheblich bezeichneten Tatsachen" auch solche Tatsachen ,als subventionserheblich im Sinne des Absatzes 1, von denen die Subventionsvergabe und die Rückforderung gesetzlich abhängig ist. Gemeint sind damit die materiellen Voraussetzungen für die Vergabe und Rückforderung. Soweit das Gesetz diese näher bestimmt [...], ist damit bereits die zur Klarheit des Subventionsvergabeverfahrens notwendige Konkretisierung erreicht. Es wäre zu aufwendig und eher verwirrend, wenn zusätzlich zu einer solchen gesetzlichen
Regelung noch eine Bezeichnung der Tatsachen als ,subventionserheblich' hinzutreten müßte". 39

Damit hat der Gesetzgeber einen Schritt in Richtung eines materiellen Begriffs der subventionserheblichen Tatsache getan. Zwar wurde die Idee einer Bestimmung der Subventionserheblichkeit nach dem Zweck der Subvention nicht umgesetzt, ${ }^{40}$ dennoch können auch solche Tatsachen subventionserheblich sein, hinsichtlich derer das Gesetz hinreichend deutlich zum Ausdruck bringt, dass die Gewährung, Rückforderung etc. von ihnen abhängig ist, ohne dies mit der ausdrücklichen Bezeichnung als ,subventionserheblich“ $\mathrm{zu}$ verbinden. ${ }^{41}$ Diese Regelung wurde insbesondere für Fälle geschaffen, in denen $\S 2$ SubvG nicht zur Anwendung kommt, also die Vergabe von Subventionen nach dem Gemeinschaftsrecht durch Stellen der EG erfolgt.

Daher sollte die ,bereits in den EG-Nomen erfolgte Benennung von Vergabevoraussetzungen als Grundlage der Pönalisierung dahingehender Täuschungshandlungen genügen". ${ }^{42}$ Auf diese Weise wird durch das Strafgesetz klargestellt, dass nur solche Täuschungshandlungen strafbar sind, die sich auf ,gesetzlich klar ausgewiesene Vergabevoraussetzungen“ beziehen. Da sich die Vorschrift spezifisch an einen konkreten Kreis von Subventionsnehmern richtet, stellt die Strafvorschrift an den Normadressaten auch keine höheren Anforderungen als andere Strafvorschriften.

Nach dieser Regelung reicht es also für die straftatbestandliche Subventionserheblichkeit aus, wenn sich dem Gesetz unmittelbar - auch unter Zuhilfenahme der üblichen Auslegungsmethoden - entnehmen lässt, unter welchen Voraussetzungen die Subvention gewährt wird. Das setzt voraus, dass „das Gesetz selbst mit hinreichender Deutlichkeit zum Ausdruck bringt, dass die Subventionierung unter der im Gesetz genannten Voraussetzung erfolgt, ohne die entsprechende Tatsache" ausdrücklich als subventionserheblich zu kennzeichnen. ${ }^{43}$

Das ist nach der Rechtsprechung des BGH nicht der Fall, wenn das Gesetz der Verwaltung einen Ermessensspielraum bei der Entscheidung über die Subvention - hinsichtlich der konkreten Tatsache - gibt. Dann sei dem Gesetz nämlich für den konkreten Einzelfall nicht zu entnehmen, ob die Bewilligung der Subvention von der Voraussetzung abhängt. Es komme vielmehr eine an den konkreten Umständen des Einzelfalls orientierte Ermessensentscheidung hinzu. Allein die Kenntnis des Gesetzes reiche in diesen Fällen nicht aus, um

\footnotetext{
${ }^{39}$ BT-Drs. 7/3441, S. 28.

${ }^{40} \mathrm{Zu}$ diesem Vorschlag BT-Drs. 7/3441, S. 4, 29; zur Kritik daran BT-Drs. 7/5291, S. 13.

${ }^{41}$ So BT-Drs. 7/5291, S. 13.

42 BT-Drs. 7/5291, S. 13.

${ }^{43}$ BGH, Beschl. v. 30.9.2010 - 5 StR 61/10 = wistra 2011, 67 (68); BGH, Urt. v. 11.11.1998 - 3 StR 101/98 = BGHSt 44, 233 (241); im Ergebnis auch BGH, Urt. v. 20.6.1986 - 1 StR 184/86 = BGHSt 34, $111 \mathrm{ff}$.
} 
im Einzelfall zu beurteilen, ob die Subventionierung an die Erfüllung der Voraussetzung geknüpft ist. ${ }^{44}$

Diese ,gesetzliche Abhängigkeit“ der Subvention von einer Tatsache kann durch formelle Bundes- und Landesgesetze, durch normative Festlegungen im Haushaltsplan und durch das Haushaltsgesetz hergestellt werden, aber natürlich auch durch die Regelungen des Unionsrechts.

Das wirft die Frage auf, ob auch die Erfüllung von CCVorgaben als eine subventionserhebliche Tatsache anzusehen ist. Bei der Entwicklung einer Antwort sind zwei Aspekte zu trennen: zum einen die Abhängigkeit der Subvention von der Erfüllung der CC-Vorgaben und zum anderen die hinreichende Bestimmtheit der CC-Vorgaben selbst im Hinblick auf Art. 103 Abs. 2 GG. Denn es ist durchaus denkbar, dass die (vollständige) Gewährung einer Subvention zweifellos von der Erfüllung der Voraussetzungen einer bestimmten Vorschrift abhängig ist, die Sanktionsvorschrift selbst aber so unbestimmt ist, dass im Ergebnis keine hinreichend bestimmte Strafvorschrift vorliegt.

\section{c) Subventionserheblichkeit von Cross-Compliance-Vorgaben}

Die Vorschriften des Unionsrechts über die Einhaltung der „,anderweitigen Verpflichtungen“ sind insofern subventionsrechtsuntypisch, als sie nicht ausdrückliche Voraussetzung für die Bewilligung oder Gewährung der Subvention sind. Es ist vielmehr geregelt, dass gegen den Subventionsnehmer, der die CC-Vorschriften gem. Art. 93 nicht erfüllt, eine sog. Verwaltungssanktion verhängt wird. Würde es sich hierbei um eine klassische Geldbuße handeln, so wäre die Nichterfüllung von CC-Auflagen zweifellos keine subventionserhebliche Tatsache, sondern eine im weitesten Sinne strafrechtliche Sanktion.

Hier ist die Situation allerdings anders, weil die angedrohte Verwaltungssanktion ausschließlich in einer Kürzung der Subvention um einen bestimmten Prozentsatz besteht, der anhand des Gesamtwerts der Direktzahlung zu ermitteln ist (Art. 99 VO [EU] Nr. 1306/2013). Die Sanktion ist damit unmittelbar mit der Gewährung der Subvention verbunden, die ihrerseits die Höhe der Sanktion begrenzt. Zudem wird in Erwägungsgrund 53 der VO (EU) Nr. 1306/2013 ausdrücklich betont, dass die ,volle Zahlung einiger GAP-Fördermittel" nur bei Erfüllung der CC-Vorgaben erfolgen soll. Daraus wird deutlich, dass die Einhaltung der CC-Verpflichtungen integraler Bestandteil des Subventionssystems ${ }^{45}$ und damit Voraussetzung für die Belassung der Subvention ist. Einer strafrechtlichen Sanktionierung neben der Subventionskürzung steht also der ne bis in idem-Grundsatz nicht entgegen, weder aus Art. 103 Abs. 3 GG, noch aus Art. 50 EU-GRCh.

Denn das Unionsrecht versteht die Verwaltungssanktion trotz ihres Abschreckungszwecks (vgl. u.a. Erwägungsgrund

\footnotetext{
${ }^{44}$ BGH, Beschl. v. 30.9.2010 - 5 StR 61/10 = wistra 2011, 67 (68); Fischer, StGB, Strafgesetzbuch und Nebengesetze, Kommentar, 66. Aufl. 2019, § 264 Rn. 17.

${ }^{45}$ Vgl. EuGH, Urt. v. 13.12.2012 - C-11/12 (Maatschap), Rn. 35 = NVwZ 2013, 134 (135).
}

19 zu VO [EU] Nr. 640/2014) - nicht als Strafe, ${ }^{46}$ sondern lässt ausdrücklich die Verhängung zusätzlicher nationaler Strafmaßnahmen zu (Art. 3, Erwägungsgrund 4 zu VO [EU] Nr. 640/2014). Ablehnung, Rücknahme und Sanktion werden als einheitliches System von Steuerungsinstrumenten begriffen (vgl. Erwägungsgrund 31 zu VO [EU] Nr. 640/2014). Daher spricht nichts dagegen, auch solche Tatsachen, von denen die Verhängung der Sanktion abhängt, als subventionserheblich anzusehen. Die Verhängung der Sanktion ist damit eine Entscheidung über das Gewähren der Subvention im Sinne von $\S 264$ Abs. 9 Nr. 2 StGB, weil damit unmittelbar eine Entscheidung über den Auszahlungsbetrag getroffen wird.

Voraussetzung für die Qualifizierung der Sanktionsvoraussetzungen als subventionserhebliche Tatsachen i.S.v. $\S 264$ Abs. 8 Nr. 2 StGB ist ferner, dass die Sanktionierung nicht vornehmlich auf einer Ermessensentscheidung der Behörde beruht. Andernfalls könnte es nämlich an der gesetzlichen Abhängigkeit der Subventionsbelassung von der konkreten Tatsache (Nichterfüllung von GAB) fehlen. Insofern kommt es auf die hinreichende Bestimmtheit an, sowohl der tatbestandlichen Voraussetzungen der Sanktion, als auch der Rechtsfolge in Form der Kürzung der Subvention.

\section{aa) Bestimmtheit des Sanktionstatbestandes}

Hier ist eine Einzelfallbetrachtung geboten. Die jeweilige CC-Vorgabe muss erkennen lassen, in welchen konkreten Fällen eine Verwaltungssanktion zu verhängen ist und ob diese Vorgabe dem Bestimmtheitsgebot gerecht wird. Betrachtet man die GAB 11 bis 13, die sich mit dem Tierschutz befassen und ihre Umsetzungen durch die TierSchNutztV, ${ }^{47}$ unter diesem Blickwinkel, so ist wird deutlich, dass zumindest diese Vorgaben hinreichend bestimmt sind und das „„Ob“ der Sanktion gerade nicht in das Ermessen der Behörde stellen. Daran ändert auch der Umstand nichts, dass in vielen Fällen, insbesondere bei Fragen der CC-Verstöße in der Tierhaltung, eine veterinärmedizinische oder ethologische Beurteilung des Sachverhalts erforderlich ist. Hierbei wird kein Ermessen ausgeübt, sondern der Sachverhalt unter den Tatbestand einer Rechtsvorschrift subsumiert.

Art. 3, 4 und der Anhang I der RL 2008/119/EG bzw. $\S \S 5$ ff. TierSchNutztV enthalten unionsrechtlich verbindliche Mindestvorgaben für die Haltung von Kälbern. Mag auch mancher Begriff dieser Regelungen auslegungsbedürftig sein oder von einer veterinärmedizinischen oder ethologischen Bewertung abhängen, so sind die Vorschriften doch so eindeutig, dass der Unternehmer erkennen kann, welche Mindestbedingungen einzuhalten sind. Für die Vorgaben zur Haltung von Schweinen nach Art. 3 und 4 und Anhang I der RL Nr. 2008/120/EG bzw. §§ 21 ff. TierSchNutztV gilt dies gleichermaßen.

\footnotetext{
${ }^{46}$ Vgl. EuGH, Urt. v. 5.6.2012 - C-489/10 (Bonda), Rn. $45=$ EuZW 2012, 543 (545).

${ }^{47}$ Tierschutz-Nutztierhaltungsverordnung in der Fassung der Bekanntmachung vom 22.8.2006, BGB1. I, S. 2043, i.d.F. v. 30.6.2017, BGBl. I, S. 2147.
} 
Missachtet ein Unternehmer in seinem Betrieb z.B. die Vorgaben aus Anhang I Kapitel 1 Allgemeine Bedingungen Ziffer 4, dass Schweine ständigen Zugang zu ausreichenden Mengen an gesundheitlich ungefährlichen Spielmaterialien haben müssen ( $\$ 26$ Abs. 1 Nr.1 TierSchNutztV), so begeht er nicht nur eine Ordnungswidrigkeit, sondern auch einen CC-Verstoß. Zwingende Rechtsfolge dieses PflichtenverstoBes - soweit er fahrlässig oder vorsätzlich erfolgt - ist die Kürzung der Subventionen nach Art. 91 ff. VO (EU) Nr. 1306/2013.

Das gilt natürlich ebenso für jede andere Verletzung von Art. 3 und 4 RL 2008/120/EG und $\S 22$ Abs. 3 TierSchNutztV, wenn also für die Schweine im Stall Verletzungsgefahr besteht, weil der Boden zu rutschig ist, sie nicht jeden Tag ausreichend gefüttert werden, nicht alle Tiere gleichzeitig Zugang zum Futter haben, die Schwänze routinemäßig kupiert werden, kein hinreichender Zugang zu Frischwasser oder Licht gegeben ist und vieles mehr.

Diese Verstöße haben nach Art. 91 VO (EU) Nr. 1306/ 2013 bei schuldhafter Begehung durch den Begünstigten eine Verwaltungssanktion zur Folge, weil es sich um Nichterfüllungen im Sinne von Art. 93 Abs. 1 lit. c i.V.m. Anhang II VO (EU) Nr. 1306/2013 handelt. Der Tatbestand der Sanktion ist damit hinreichend klar bestimmt und auch diese Voraussetzung des $§ 264$ Abs. 9 Nr. 2 StGB erfüllt.

\section{bb) Bestimmtheit der Sanktionsfolge}

Die Bestimmtheit des Sanktionstatbestandes allein reicht jedoch nicht aus, um die Eigenschaft des CC-Verstoßes als subventionserhebliche Tatsache im Sinne von $§ 264$ Abs. 9 Nr. 2 StGB zu begründen. Die Subventionserheblichkeit des Umstandes - also einschließlich seiner Rechtsfolge der Nichtgewährung oder Rückforderung der Subvention - muss sich vielmehr unmittelbar aus dem Gesetz ergeben. Jedoch ist auch das vorliegend gegeben:

Bei der Verhängung der Verwaltungssanktion hat die Behörde zwar einen gewissen Entscheidungsspielraum, Entschließungsermessen kommt ihr aber nicht zu (gebundene Entscheidung), ${ }^{48}$ weil nur ausnahmsweise von einer Kürzung der Subvention abgesehen werden darf (Art. 91 Abs. 2 UAbs. 1 VO [EU] Nr. 1306/2013) und die Grenzen, in denen sich die Sanktion zu bewegen hat, durch europäisches Verordnungsrecht vorgegeben ist. Die unmittelbare Abhängigkeit der Subvention von der gesetzlichen Vorgabe wird durch die mögliche Spannbreite der Sanktionshöhe und die Notwendigkeit einer Einzelfallbetrachtung nicht in Frage gestellt. Art. 99 VO (EU) Nr. 1306/2013 enthält detaillierte Vorgaben für die Höhe der Kürzungen. Aus dem Unionsrecht ergibt sich damit unmittelbar, dass die Tatsache des CC-Verstoßes für die Höhe der Subvention relevant ist und in welchem Rahmen es zur Kürzung kommen würde.

Ist dem Unternehmer beispielsweise bei Antragstellung bekannt, dass er in seinem Betrieb die Vorgaben der GAB 11-13 zum Tierschutz, aus baulichen, wirtschaftlichen oder

${ }^{48}$ OVG Greifswald, Beschl. v. 28.4.2009 - 2 L 171/07 = RdL (Recht der Landwirtschaft) 2009, 334 f.; ferner Booth (Fn. 3), Rn. 222. sonstigen Gründen nicht erfüllt oder zu irgendeinem Zeitpunkt im Antragsjahr nicht wird erfüllen können, so handelt es sich um einen vorsätzlichen CC-Verstoß. Dieser ist nach Art. 99 Abs. 3 VO (EU) Nr. 1306/2013 mit einer Kürzung der Direktzahlung von mindestens $20 \%$ zu ahnden. Mit Blick auf diese strikte Mindestsanktionsregelung ist zumindest in Vorsatzfällen von einer so hinreichend bestimmten Rechtsfolge auszugehen, dass sich die Subventionserheblichkeit eines Verstoßes unmittelbar aus dem Gesetz - hier den Unionsverordnungen - ergibt. Aufgrund dieser klaren Regelungen aus dem Unionsrecht, die die Berechnungen der Subventionen vorgeben, ist die Feststellung des konkreten Kürzungsbetrages in der Praxis auch automatisiert und erfolgt durch eine entsprechende Software, die die Berechnungen vornimmt. Auch die Rechtsfolge ist damit konkret vorhersehbar und der Antragsteller kann sich ohne weiteres darauf einstellen, welche Folgen unrichtige oder unvollständige Angaben haben werden.

cc) Unionsrechtskonforme Auslegung von $\$ 264$ Abs. 9 Nr. 2 StGB

Diese Interpretation von $\S 264$ Abs. 9 Nr. 2 StGB ist auch mit Blick auf die Vorgaben des Unionsrechts geboten. Bereits die VO (EG/Euratom) Nr. 2988/95 vom 18.12.1995 über den Schutz der finanziellen Interessen der EG ließ in ihren Erwägungsgründen keinen Zweifel daran, dass zu den zu schützenden Finanzmitteln insbesondere die Mittel der gemeinsamen Agrarpolitik gehören. ${ }^{49}$

Damit gilt für die Bekämpfung von Missbrauchshandlungen im Zusammenhang mit Agrarsubventionen, was der EuGH in seiner Rechtsprechung zum Mehrwertsteuerrecht stets hervorhebt: Die Mitgliedstaaten haben dafür zu sorgen, dass die Haushaltsmittel der EU vor Betrugshandlungen im weitesten Sinne zum einen durch effektive, abschreckende und angemessene Sanktionen geschützt werden. Zum anderen sind Betrugstaten zum Nachteil der Union ebenso effektiv zu verfolgen, wie Straftaten gegen die finanziellen Interessen des jeweiligen Mitgliedstaats. ${ }^{50}$

Das führt dazu, dass Strafvorschriften, die im konkreten Fall den Schutz der finanziellen Interessen bezwecken, im Rahmen des unionsrechtlichen Anwendungsvorrangs und der Charta der Europäischen Grundrechte so auszulegen sind, dass sie einen effektiven und äquivalenten Schutz des europäischen Agrarhaushalts gewährleisten. Wollte man § 264 Abs. 9 Nr. 2 StGB so interpretieren, dass die Umstände, die zur Kürzung der Direktzahlungen führen können, keine subventionserhebliche Tatsache sind, so wäre die Effektivität des Schutzes der Agrarbeihilfen vor Betrugshandlungen - im weiten unionsrechtlichen Sinn - erheblich gemindert. Daher gebietet die unionsrechtskonforme Auslegung von $\S 264$ Abs. 9 Nr. 2 StGB die Qualifizierung von CC-Verstößen als subventionserhebliche Tatsachen.

\footnotetext{
${ }^{49}$ Vgl. auch EuGH, Urt. v. 5.6.2012 - C-489/10 (Bonda) = EuZW 2012, 543 ff.; BT-Drs. 19/7886, S. 8.

${ }^{50}$ Vgl. nur EuGH, Urt. v. 5.12.2017 - C-42/17 (M.A.S \& M.B), Rn. 30.
} 


\section{Machen unrichtiger oder unvollständiger Angaben}

Die Tatsachen, die zu einer Kürzung der Subvention im Sanktionswege führen, sind also subventionserheblich. Doch liegt auch eine taugliche Tathandlung vor, wenn der Unternehmer bei der Antragstellung Umstände verschweigt, deren Offenbarung zu einer zwingenden Kürzung führen würde?

Nach $\S 264$ Abs. 1 Nr. 1 StGB macht sich strafbar, wer in einem Subventionsverfahren gegenüber einer für die Bewilligung einer Subvention zuständigen Behörde oder Stelle über subventionserhebliche Tatsachen unrichtige oder unvollständige Angaben macht, die für ihn oder einen anderen vorteilhaft sind. Nach $\S 264$ Abs. 1 Nr. 3 StGB ist strafbar, wer den Subventionsgeber entgegen den Rechtsvorschriften über die Subventionsvergabe über subventionserhebliche Tatsachen in Unkenntnis lässt.

Wenn der Unternehmer über die Nichterfüllung der CCVorgaben zum Tierschutz, Einsatz von Düngemitteln etc. schweigt, scheint zunächst nur pflichtwidriges Verschweigen von subventionserheblichen Tatsachen in Betracht zu kommen, weil über die relevanten Umstände keine Angaben gemacht werden. Eine solche Bewertung griffe allerdings dann zu kurz, wenn der Antragsteller wie in Ziffer 6 im „Sammelantrag 2019 Anlage A Auszahlungsantrag Basisprämie und Greeningprämie“ (NRW) erklärt, dass er sich verpflichtet, ,die Bestimmungen der Verordnungen des Europäischen Parlaments und des Rates und der Kommission der Europäischen Union und des Bundes zu den EU-Prämien in den jeweils geltenden Fassungen einzuhalten $[\ldots]^{\text {“. Es }}$ folgt eine Aufzählung der maßgeblichen EU-Verordnungen und Bundesgesetze.

Diese Erklärung ist nach dem objektiven Empfängerhorizont auszulegen und daher nicht nur als Verpflichtungserklärung zu verstehen, sondern auch als Erklärung, dass dem Antragsteller keine Umstände bekannt sind, die einer Erfüllung dieser Verpflichtung zwingend entgegenstehen. Wer sich bereit erklärt eine Pflicht einzuhalten, versichert damit konkludent, dass ihm die Einhaltung dieser Pflicht (seines Wissens nach) auch möglich ist. Wer einen Antrag auf Bewilligung der Basisprämie stellt, obwohl er weiß, dass die Voraussetzungen für die Gewährung der Subvention in voller Höhe nicht gegeben sind, macht also zumindest unvollständige Angaben im Sinne von $\S 264$ Abs. 1 Nr. 1 StGB, begeht mithin eine taugliche Subventionsbetrugshandlung.

Denn unvollständige Angaben i.S.v. § 264 Abs. 1 Nr. 1 StGB macht, wer durch ausdrückliche oder konkludente Gedankenerklärungen $^{51}$ über Tatsachen mit Blick auf die Bedingungen der Subventionserteilung ein unvollständiges oder unrichtiges und für ihn vorteilhaftes Gesamtbild vermittelt. ${ }^{52}$ Diese Voraussetzungen sind hier erfüllt.

\section{Vorsatz oder Leichtfertigkeit}

Die Strafbarkeit der Tathandlung setzt beim abstrakten Gefährdungsdelikt des Subventionsbetrugs nicht den Eintritt

\footnotetext{
51 Vgl. nur Straßer, in: Graf/Jäger/Wittig (Fn. 31), § 264 Rn. 57 f.

${ }^{52}$ Vgl. Fischer (Fn. 44), § 264 Rn. 22 ff.
}

eines Vermögensschadens voraus, sondern neben der Tathandlung nur, dass der Täter die Tathandlung vorsätzlich (§§ 264, 15 StGB) oder leichtfertig (vgl. § 264 Abs. 5 StGB) begangen hat.

\section{a) Vorsätzlicher Subventionsbetrug}

Vorsatz bedeutet vorliegend, dass der Antragsteller zumindest ernsthaft für möglich hält, dass die Tierhaltung, Verwendung von Dünger oder Giftstoffen in seinem Betrieb nicht den CC-Vorgaben entspricht, sich aber damit abfindet, z.B. weil ihm die Einhaltung der Vorgaben zu aufwendig erscheint oder er damit überfordert ist. ${ }^{53}$

Der Vorsatz muss sich dabei auch auf die Möglichkeit der Subventionskürzung beziehen, weil nur dann auch hinsichtlich der Subventionserheblichkeit vorsätzliches Handeln gegeben ist. Wer nicht weiß, dass sein Handeln zu einer Subventionskürzung führen kann, kennt die Subventionserheblichkeit der Tatsachen nicht, zu denen er Angaben macht. Daher muss der Unternehmer zum einen wissen, dass sein Handeln (möglicherweise) nicht den CC-Vorgaben entspricht und dass sich daraus die Kürzung der Subvention ergeben kann. Er muss jedoch nicht die konkreten Vorschriften kennen, gegen die er möglicherweise verstößt und aufgrund derer die Kürzung erfolgt. Erkennt er die Möglichkeit, aufgrund eines Fehlverhaltens mit einer Sanktion belegt zu werden, so handelt er mit Blick auf die Subventionserheblichkeit vorsätzlich.

Damit begeht der Unternehmer eine vorsätzliche Tathandlung, der die baulichen Gegebenheiten seines Betriebes kennt und weiß, dass die Spalten in den Betonböden der Schweineställe unzulässig breit sind ${ }^{54}$ und sich bewusst ist, dass dies zu einer Kürzung der Subventionen führen könnte, aber aus wirtschaftlichen Gründen keine Änderungen vornimmt. Soweit es die mangelnde Kenntnis der relevanten Bestimmungen über die CC-Vorgaben angeht, wird dem Antragsteller eine Berufung auf mögliches Nichtwissen dadurch erschwert, dass er unter Ziffer 4 der Anlage A zum Sammelantrag 2019 (Basisprämie/Greeningprämie) versichern muss, dass ihm die maßgeblichen Vorschriften des Unionsrechts in den jeweiligen Fassungen bekannt sind.

\section{b) Leichtfertiger Subventionsbetrug}

Der Subventionsbetrug in den Fällen des $§ 264$ Abs. 1 Nr. 1 bis 3 StGB ist nach $\S 264$ Abs. 5 StGB auch bei leichtfertiger Begehung strafbar. Leichtfertigkeit ist eine besonders schwerwiegende Form der Fahrlässigkeit. Sie wird vom BGH als „vorsatznahe Schuldform“ angesehen und setzt eine be-

\footnotetext{
${ }^{53}$ Grundlegend zu den Voraussetzungen des Eventualvorsatzes BGH, Urt. v. 22.4.1955 - 5 StR 35/55 = BGHSt 7, 363 (368 ff.).

${ }^{54}$ Art. 3 Abs. 2 lit. b RL 2008/120/EG schreibt zum Schutz von Schweinen vor Verletzungen vor, dass die Spalten in Betonspaltenböden, die insbesondere zu leichteren Reinigung der Ställe verwendet werden, nicht breiter sein dürfen als z.B. $14 \mathrm{~mm}$ bei Mastferkeln und $18 \mathrm{~mm}$ bei Mastschweinen.
} 
sondere Gleichgültigkeit oder grobe Unachtsamkeit voraus. ${ }^{55}$ Der Täter muss nach seinen individuellen Fähigkeiten ${ }^{56}$ in der Lage gewesen sein, die Subventionserheblichkeit und die für ihn gebotene Handlungsweise - hier: die Offenlegung des CC-Verstoßes - ohne weiteres $\mathrm{zu}$ erkennen. ${ }^{57}$ Leichtfertig handelt regelmäßig, wer sich um die Vergabevoraussetzungen nicht oder nur oberflächlich kümmert, sich über die Vollständigkeit oder Richtigkeit seiner Angaben keine Gedanken macht oder die Angaben eines unzuverlässigen oder unerprobten Mitarbeiters ungeprüft übernimmt. ${ }^{58}$

Im vorliegenden Zusammenhang ist zu berücksichtigen, dass auch die Leichtfertigkeit sich auf die beiden oben bereits genannten Aspekte beziehen muss: Der Täter muss zum einen die Umstände grob verkennen, die einen CC-Verstoß begründen. Dies gilt etwa, wenn er nicht weiß, wie breit die Spalten in den Betonböden in seinem Betrieb sind oder ihm unbekannt ist, dass die tatsächliche Breite unzulässig ist. Denn in diesem Fall ist von einem besonders groben Verstoß gegen die Informationspflichten des Tierhalters auszugehen. Zum einen ergibt sich aus $\S 2$ Nr. 3 TierSchG unmittelbar, dass sich jeder Tierhalter alle erforderlichen Informationen beschaffen muss, die er benötigt, um ein Tier artgerecht und legal zu halten. Zum anderen wird in den Antragsunterlagen ausdrücklich auf die Geltung der Vorgaben der RL 2008/120/ EG hingewiesen. Der Antragsteller muss zudem versichern, dass ihm die relevanten Vorschriften bekannt sind. Gibt ein Unternehmer diese Erklärung $a b$, so dürfte er sich später kaum erfolgreich darauf berufen können, die Rechtslage nicht gekannt und daher nicht leichtfertig gehandelt zu haben. Dies gilt insbesondere deswegen, weil die für die Bewilligung zuständigen Behörden umfangreiche Beratungen $\mathrm{zu}$ allen relevanten Fragen anbieten.

\section{Strafrechtliche Relevanz von Cross-Compliance Ver- stößen}

Der Blick auf die Gesamtsituation der Gewährung von Direktzahlungen aus dem Agrarhaushalt der Union und des Kürzungsregimes der Art. 91 ff. VO (EU) Nr. 1306/2013 macht deutlich, dass bei der Nichterfüllung von CC-Verpflichtungen neben die Kürzung von Subventionen im Sanktionswege die Strafbarkeit wegen Subventionsbetruges nach $\S 264$ StGB treten kann.

Erklärt ein Antragsteller, er halte die ,anderweitigen Verpflichtungen“ ein, obwohl er weiß oder damit rechnet, dass er

${ }^{55}$ BGH, Urt. v. 17.7.1997 - 1 StR 791/96 = BGHSt 43, 158 (167); BGH, Beschl. v. 20.5.2010 - 5 StR 138/10 = NStZ-RR 2010, 311 (312); BGH, Urt. v. 13.12.2012 - 5 StR 542/12 = wistra 2013, 149; Hellmann, in: Kindhäuser/Neumann/ Paeffgen (Hrsg.), Nomos Kommentar, Strafgesetzbuch, Bd. 3, 5. Aufl. 2017, § 264 Rn. 153.

${ }^{56}$ Hierzu BGH, Urt. v. 24.1.2006 - 1 StR 357/05 = BGHSt 50, 347 (352).

${ }^{57}$ BGH, Beschl. v. 13.12.2012 - 5 StR 542/12 = NStZ 2013, 406.

58 Fischer (Fn. 44), § 264 Rn. 37; Tiedemann, in: Jähnke/ Laufhütte/Odersky (Hrsg.), Strafgesetzbuch, Leipziger Kommentar, Bd. 7, 11. Aufl. 2005, § 264 Rn. 145. dazu nicht in der Lage ist oder sie bereits verletzt hat bzw. haben könnte, so liegt eine Strafbarkeit nach § 264 Abs. 1 Nr. 1 StGB nahe. Sicheres Wissen um den Verstoß ist hier ebenso wenig erforderlich, wie um die Sanktionsfolge. Die Nichterfüllung der CC-Vorgaben ist aufgrund der zwingenden Verhängung der Kürzungssanktion eine sich unmittelbar aus dem Unionsrecht ergebende subventionserhebliche Tatsache.

Selbst wenn der Agrarunternehmer nicht einmal die Möglichkeit erkannt hat, dass seine Angaben im Hinblick auf die Subventionserheblichkeit unvollständig oder unrichtig sind, kommt eine Strafbarkeit in Betracht, weil auch die leichtfertige Tatbegehung strafbar ist.

Das führt im Ergebnis dazu, dass die unterlassene Offenbarung von systematischen Verstößen gegen CC-Vorgaben im Antrag auf Bewilligung und Auszahlung von Direktzahlungen typischerweise auch eine Straftat nach § 264 Abs. 1 oder Abs. 5 StGB darstellt. Denn der Agrarunternehmer, der im Mai seinen Sammelantrag mit den entsprechenden Anlagen abgibt und die Einhaltung der CC-Vorgaben zu den GAB versichert, weiß in diesem Zeitpunkt regelmäßig, unter welchen Bedingungen er in dem betreffenden Jahr bislang seine Tiere gehalten, wie er Düngemittel verwendet und ob er Hecken oder Teiche beseitigt hat. 